\title{
FUNCTIONS WITH A CONCAVE MODULUS OF CONTINUITY
}

\author{
H. E. WHITE, JR.
}

\begin{abstract}
In [1], C. Goffman proved that, if $\sigma$ is a modulus of continuity, then the set of all functions $f$ in $C[0,1]$ such that $m(\{x: f(x)=g(x)\})=0$ ( $m$ denotes Lebesgue measure) for all $g$ in $C(\sigma)$, the set of all functions in $C[0,1]$ having $\sigma$ as a modulus of continuity, is residual in $C[0,1]$. In the present article, we prove that, if $\sigma$ is a concave modulus of continuity and $0<K<24^{-1}$, then the set of all functions $f$ in $C(\sigma)$ such that $m(\{x: f(x)=g(x)\})=0$ for all $g$ in $C(K \sigma)$ is residual in $C(\sigma)$. Using this result, we show that, if $0<\alpha<1$, then there are functions in $C[0,1]$ which satisfy a Hölder condition of exponent $\alpha$ such that $m(\{x: f(x)=g(x)\})=0$ for all $g$ in $C[0,1]$ which satisfy a Hölder condition of exponent $>\alpha$.
\end{abstract}

1. By a modulus of continuity we mean a real valued function $\sigma$ defined on $[0,1]$ such that: (1) if $0<x \leqq y \leqq 1$, then $0<\sigma(x) \leqq \sigma(y)$, (2) $\sigma$ is continuous at 0 , and (3) $\sigma(0)=0$. We say that a function $f$ in $C[0,1]$ has modulus of continuity $\sigma$ if

$$
|f(y)-f(x)| \leqq \sigma(|y-x|)
$$

for all $x, y$ in $[0,1]$. Here $C[0,1]$ denotes the set of all continuous, real valued functions defined on $[0,1]$. We denote by $C(\sigma)$ the set of all functions in $C[0,1]$ having modulus of continuity $\sigma$.

In [1], C. Goffman proved the following statement.

1.1 THEOREM. If $\sigma$ is a modulus of continuity, then the set of all functions $f$ in $C[0,1]$ such that $\{x: f(x)=g(x)\}$ has Lebesgue measure 0 for all $g$ in $C(\sigma)$ is residual in $C[0,1]$ (i.e. its complement in $C[0,1]$ is of the first category in $C[0,1])$.

This result leads naturally to the following question. If $\sigma$ and $\mu$ are moduli of continuity, under what conditions is $U(\sigma, \mu)$, the set of all functions $f$ in $C(\sigma)$ such that $m(\{x: f(x)=g(x)\})=0$ for all $g$ in $C(\mu)$, residual in $C(\sigma)$ ? Here $m$ denotes Lebesgue measure and $C(\sigma)$ is equipped with the restriction of the usual metric on $C[0,1]$.

Presented to the Society, August 31, 1972; received by the editors January 15, 1972. AMS (MOS) subject classifications (1970). Primary 26A15, 26A16.

Key words and phrases. Modulus of continuity, Hölder condition, concave modulus of continuity.

(c) American Mathematical Society 1974 
In this paper we prove the following statement.

1.2 TheOREM. If $\sigma$ is a concave modulus of continuity and

$$
\lim _{x \rightarrow 0} \sup \left[\mu(x) \sigma^{-1}(x)\right]<24^{-1},
$$

then $U(\sigma, \mu)$ is residual in $C(\sigma)$.

The following corollary to 1.2 answers in the affirmative a question which Dr. Goffman suggested.

1.3 CoRollary. If $0<\alpha<1$, then there is a function $f$ in $C[0,1]$ such that: (1) $f$ satisfies a Hölder condition of exponent $\alpha$, and (2) if $g$ in $C[0,1]$ satisfies a Hölder condition of exponent $\beta>\alpha$, then $m(\{x: f(x)=g(x)\})=0$.

2. If $\sigma$ is a modulus of continuity and $A \subset[0,1]$, we denote by $C(A, \sigma)$ the set of all functions $f: A \rightarrow R$ for which (1.1) holds for all $x, y$ in $A$. We denote by $\operatorname{Lip}(A, \sigma)$ the set $\bigcup\{C(A, M \sigma): M>0\}$. If $f \in \operatorname{Lip}(A, \sigma)$, we let

Then

$$
\|f\|_{\sigma}=\inf \{M>0: f \in C(A, M \sigma)\} \text {. }
$$

$$
|f(y)-f(x)| \leqq\|f\|_{\sigma} \sigma(|y-x|)
$$

for all $x, y$ in $A$. We will denote $\operatorname{Lip}([0,1], \sigma)$ by $\operatorname{Lip}(\sigma)$. As usual, for $f$ in $C[0,1],\|f\|$ denotes $\max \{|f(x)|: x \in[0,1]\}$.

A modulus of continuity $\sigma$ is called subadditive if $\sigma(x+y) \leqq \sigma(x)+\sigma(y)$ for all $x, y$ in $[0,1]$ such that $x+y \in[0,1]$. A modulus of continuity $\sigma$ is called concave if, for any $x, y$ in $[0,1]$ such that $x<y$, we have $\sigma(t) \geqq$ $T(t)$ for all $t$ in $[x, y]$, where $T$ is the linear function such that $T(x)=\sigma(x)$ and $T(y)=\sigma(y)$.

In the rest of this paper, $\sigma$ will always denote a concave modulus of continuity and $J$ will always denote a closed subinterval of $[0,1]$. We denote the set of all nonnegative integers by $N$. For $i, j$ in $N$, let $N(i, j)=$ $\{k \in N: i \leqq k \leqq j\}$ and $N(j)=N(0, j)$. Let $N^{+}=N-\{0\}$.

Since quasi-linear functions play an important role in the proof of Theorem 1.2, it is convenient to have the following terminology. By a quasi-linear function on $J$ we mean a function $\varphi$, defined and continuous on $J$, such that there is a partition $P=\left\{x_{j}: j \in N(n)\right\}$ of $J$ for which $\varphi \mid\left[x_{j}, x_{j+1}\right]$ is linear for all $j$ in $N(n-1)$. In this case, $P$ is called a $\varphi$ partition of $J$. If $P=\left\{x_{j}: j \in N(n)\right\}$ is a partition of $J$ and $\left(c_{j}\right)_{j \in N(n)}$ is a family of real numbers, then the quasi-linear function $\varphi$ defined on $J$ such that $P$ is a $\varphi$-partition of $J$ and $\varphi\left(x_{i}\right)=c_{i}$ for $i \in N(n)$ is called the quasilinear function determined by $\left\{\left(x_{j}, c_{j}\right): j \in N(n)\right\}$. For any partition $P=$ $\left\{x_{j}: j \in N(n)\right\}$ of $J$, we denote by $\|P\|$ the number $\max \left\{x_{j+1}-x_{j}: j \in N(n-1)\right\}$. Finally, the set of all quasi-linear functions defined on $J$ is denoted by $Q(J)$. 
3. We shall prove 1.2 via a sequence of lemmata.

3.1 Lemma. Suppose that $P=\left\{x_{j}: j \in N(n)\right\}$ is a partition of $J$ and that $\left(c_{j}\right)_{j \in N(n)}$ is a family of real numbers such that

$$
\left|c_{j}-c_{i}\right| \leqq \sigma\left(\left|x_{j}-x_{i}\right|\right)
$$

for all $i, j$ in $N(n)$. Let $\varphi$ denote the quasi-linear function determined by $\left\{\left(x_{j}, c_{j}\right): j \in N(n)\right\}$. Then $\varphi \in C(J, \sigma)$.

Proof. Suppose $x, y \in J$. We may assume $\{x, y\} \cap P \neq \varnothing$. [If $\{x, y\} \cap$ $P=\varnothing$, then there are $x^{\prime}, y^{\prime}$ in $J$ such that $\left|y^{\prime}-x^{\prime}\right|=|y-x|,\left|\varphi\left(y^{\prime}\right)-\varphi\left(x^{\prime}\right)\right| \geqq$ $|\varphi(y)-\varphi(x)|$, and $\left\{x^{\prime}, y^{\prime}\right\} \cap P \neq \varnothing$.] Suppose $x<y, x=x_{i}$, and $k$ in $N(n)$ is such that $x_{k} \leqq y \leqq x_{k+1}$. Then, since (3.1) holds, $\varphi\left(x_{k}\right)-\varphi\left(x_{i}\right) \leqq \sigma\left(x_{k}-x_{i}\right)$ and $\varphi\left(x_{k+1}\right)-\varphi\left(x_{i}\right) \leqq \sigma\left(x_{k+1}-x_{i}\right)$. Since $\varphi$ is linear on $\left[x_{k}, x_{k+1}\right]$ and $\sigma$ is concave, $\varphi(y)-\varphi\left(x_{i}\right) \leqq \sigma\left(y-x_{i}\right)$. Similarly, $\varphi(y)-\varphi\left(x_{i}\right) \geqq-\sigma\left(y-x_{i}\right)$.

3.2 Corollary. Suppose $\varphi \in Q(J)$ and $P=\left\{x_{j}: j \in N(n)\right\}$ is a $\varphi$ partition of $J$. Then

Proof. Let $M$ denote the number on the right hand side of (3.2). Applying 3.1, with $c_{j}=\varphi\left(x_{j}\right)$ for $j \in N(n)$, we have $\|\varphi\|_{\sigma} \leqq M$. By (2.1), $\|\varphi\|_{\sigma} \geqq M$.

\subsection{Corollary. $Q(J) \cap C(J, \sigma)$ is dense in $C(J, \sigma)$.}

3.4 Lemma. Suppose $\varphi$ is a linear function defined on $J=[a, b]$. If $x, y \in J$ and $|y-x| \geqq \frac{1}{4}(b-a)$, then

$$
|\varphi(y)-\varphi(x)| \geqq \frac{1}{4}\|\varphi\|_{\sigma} \sigma(|y-x|) .
$$

Proof. If $x, y$ are as hypothesized, then, using (3.2),

$$
\begin{aligned}
|\varphi(y)-\varphi(x)| & =\frac{|\varphi(b)-\varphi(a)|}{\sigma(b-a)} \frac{\sigma(b-a)}{\sigma(|y-x|)} \frac{|y-x|}{b-a} \sigma(|y-x|) \\
& \geqq 1\|\varphi\|_{\sigma} \sigma(|y-x|) .
\end{aligned}
$$

Notation. For $x$ in $[0,1]$ and $\delta>0$, let $I(x, \delta)=\left[x+\frac{1}{4} \delta, x+\frac{1}{2} \delta\right]$ and $J(x, \delta)=\left[x-\frac{1}{2} \delta, x-\frac{1}{4} \delta\right]$.

3.5 Lemma. Suppose $h>0,0<L<(24)^{-1}$, and $J=[a, b]$. There is $\theta$ in $Q(J)$ such that: (1) $\theta(a)=\theta(b)=0,(2) 0 \leqq \theta(x) \leqq h$ for all $x$ in $J,(3)\|\theta\|_{\sigma} \leqq$ $1-4 L$, and (4) there is $\delta=\delta(\theta)>0$ such that, if $x \in J$, then

$$
|\theta(y)-\theta(x)| \geqq 5 L \sigma(|y-x|)
$$

holds either for all $y$ in $I(x, \delta)$ or for all $y$ in $J(x, \delta)$. 
Proof. Choose positive numbers $d, h^{\prime}$ so that $\frac{1}{2}(b-a) d^{-1} \in N, h^{\prime} \leqq h$, and

$$
20 L \leqq h^{\prime}[\sigma(d)]^{-1} \leqq 1-4 L .
$$

Let $k=\frac{1}{2}(b-a) d^{-1}, x_{j}=a+j d$ for $j \in N(2 k), P=\left\{x_{j}: j \in N(2 k)\right\}$, and $\theta$ be the quasi-linear function determined by

$$
\left\{\left(x_{2 j}, 0\right): j \in N(k)\right\} \cup\left\{\left(x_{2 j+1}, h^{\prime}\right): j \in N(k-1)\right\} .
$$

Then, by (3.2),

$$
\begin{aligned}
\|\theta\|_{\sigma} & =\max \left\{\left|\theta\left(x_{j}\right)-\theta\left(x_{i}\right)\right|\left[\sigma\left(\left|x_{j}-x_{i}\right|\right)\right]^{-1}: i, j \in N(2 k), i \neq j\right\} \\
& =\left|\theta\left(x_{1}\right)-\theta\left(x_{0}\right)\right|\left[\sigma\left(x_{1}-x_{0}\right)\right]^{-1}=h^{\prime}[\sigma(d)]^{-1} \leqq 1-4 L .
\end{aligned}
$$

And, by (3.3), if $x, y \in\left[x_{j}, x_{j+1}\right]$ and $|y-x| \geqq \frac{1}{4} d$, then

$$
\begin{aligned}
|\theta(y)-\theta(x)| & \geqq \frac{1}{4}\left\|\theta \mid\left[x_{j}, x_{j+1}\right]\right\|_{\sigma} \sigma(|y-x|) \\
& =\frac{1}{4} h^{\prime}[\sigma(d)]^{-1} \sigma(|y-x|) \\
& \geqq 5 L \sigma(|y-x|) .
\end{aligned}
$$

Let $\delta=\frac{1}{4} d$. If $x \in\left[x_{j}, x_{j}+2 \delta\right]$, then $I(x, \delta) \subset\left[x_{j}, x_{j+1}\right]$. Hence (3.4) holds for all $y$ in $I(x, \delta)$. Similarly, if $x \in\left[x,+2 \delta, x_{j+1}\right]$, then (3.4) holds for all $y$ in $J(x, \delta)$.

For $n$ in $N^{+}$and any positive numbers $L, \eta$ such that $\eta<n^{-1}$, let $V(\sigma, L, n, \eta)$ denote the set of all $f$ in $C(\sigma)$ for which there is a measurable subset $A=A(f)$ of $[0,1]$ such that $m(A)>1-n^{-1}$ and, if $x \in A$, then there is $\delta=\delta(x)>0$ such that $\eta<\delta<n^{-1}$ and

$$
|f(y)-f(x)| \geqq L \sigma(|y-x|)
$$

holds either for all $y$ in $I(x, \delta)$ or for all $y$ in $J(x, \delta)$. Let

$$
V(\sigma, L, n)=\bigcup\left\{V(\sigma, L, n, \eta): 0<\eta<n^{-1}\right\} .
$$

3.6 Lemma. Suppose $0<L<24^{-1}, 0<\alpha<1, \varepsilon>0$, and $n \in N^{+}$. If $\varphi \in C(\alpha \sigma) \cap Q([0,1])$, then there is $\psi$ in $C(\sigma) \cap Q([0,1])$ and $\eta>0$ such that $\psi \in V(\sigma, L, n, \eta)$ and $\|\varphi-\psi\| \leqq \varepsilon$.

Proof. Let $P=\left\{x_{j}: j \in N(q)\right\}$ be a $\varphi$-partition of $[0,1]$ such that $\|P\|<n^{-1}$ and, for $j \in N(q-1)$,

$$
\left|\varphi\left(x_{j+1}\right)-\varphi\left(x_{j}\right)\right|<\frac{1}{2} \varepsilon .
$$

For $j$ in $N(q-1)$, let $I_{j}=\left[x_{j}, x_{j+1}\right], I_{j}^{\prime}=\left[x_{0}, x_{j}\right]$, and $I_{j}^{\prime \prime}=\left[x_{j+1}, x_{q}\right]$.

Suppose, for some $j$ in $N(q-1)$, we have defined $\psi$ on $I_{j}^{\prime}$ so that: (j.1) $\psi \in Q\left(I_{j}^{\prime}\right),(j .2)\left\|\psi-\varphi \mid I_{j}^{\prime}\right\| \leqq \varepsilon,(j .3) \psi\left(x_{i}\right)=\varphi\left(x_{i}\right)$ for all $i$ in $N(j)$, (j.4) if $\lambda_{j}$ is defined by letting

$$
\begin{aligned}
\lambda_{j}(x)=\psi(x) & \text { if } x \in I_{j}^{\prime}, \\
& =\varphi(x) \quad \text { if } x \in I_{j} \cup I_{j}^{\prime \prime},
\end{aligned}
$$


then $\left\|\lambda_{j}\right\|_{\sigma}<1$, and (j.5) there are a measurable subset $A_{j}$ of $I_{j}^{\prime}$ and $\eta_{j}>0$ such that $m\left(A_{j}\right)>m\left(I_{j}^{\prime}\right)-j(q n)^{-1}$ and, if $x \in A_{j}$, then there is a $\delta$ such that $\eta_{j}<\delta<n^{-1}$ and (3.5), with $f$ replaced by $\psi$, holds either for all $y$ in $I(x, \delta)$ or for all $y$ in $J(x, \delta)$.

Case 1. Suppose $\left|\varphi\left(x_{j+1}\right)-\varphi\left(x_{j}\right)\right| \geqq 4 L \sigma\left(x_{j+1}-x_{j}\right)$. Then $\left\|\varphi \mid I_{j}\right\|_{\sigma} \geqq 4 L$. Let $\psi(x)=\varphi(x)$ for all $x$ in $I_{j}$. Clearly $(j+1) \cdot 1-(j+1) .4$ are satisfied. Let $A_{j+1}=A_{j} \cup I_{j}$ and $\eta_{j+1}=\min \left(\eta_{j}, \frac{1}{4}\left(x_{j+1}-x_{j}\right)\right)$. Then $(j+1) .5$ holds, since, if $x, y \in I_{j}$ and $|y-x| \geqq \frac{1}{4}\left(x_{j+1}-x_{j}\right)$, by 3.4 ,

$$
|\varphi(y)-\varphi(x)| \geqq \frac{1}{4}\left\|\varphi \mid I_{j}\right\|_{\sigma} \sigma(|y-x|) \geqq L \sigma(|y-x|) .
$$

Case 2. Suppose $\left|\varphi\left(x_{j+1}\right)-\varphi\left(x_{j}\right)\right|<4 L \sigma\left(x_{j+1}-x_{j}\right)$. Assume that $\varphi\left(x_{j+1}\right) \geqq \varphi\left(x_{j}\right)$. By $(j .1)$, there is a $\psi$-partition $P_{1}=\left\{y_{i}: i \in N(p)\right\}$ of $I_{j}^{\prime}$. Let $d=x_{j+1}-x_{j}, x_{j}^{*}=x_{j}+\frac{1}{2} d$, and

$$
\begin{aligned}
b_{j}= & \min \left[\left\{\psi\left(y_{i}\right)+\sigma\left(x_{j}^{*}-y_{i}\right): i \in N(p)\right\}\right. \\
& \left.\cup\left\{\varphi\left(x_{i}\right)+\sigma\left(x_{i}-x_{j}^{*}\right): i \in N(j+1, q)\right\}\right] .
\end{aligned}
$$

Then $b_{j}>\varphi\left(x_{j}^{*}\right)$ since $\left\|\lambda_{j}\right\|_{\sigma}<1$. Let $a_{j}$ be a number such that

$$
\varphi\left(x_{j}^{*}\right)<a_{j}<\min \left(b_{j}, \varphi\left(x_{j+1}\right)+\frac{1}{2} \varepsilon\right)
$$

and let $\gamma$ denote the quasi-linear function determined by

$$
\left\{\left(y_{i}, \psi\left(y_{i}\right)\right): i \in N(p)\right\} \cup\left\{\left(x_{j}^{*}, a_{j}\right)\right\} \cup\left\{\left(x_{i}, \varphi\left(x_{i}\right)\right): i \in N(j+1, q)\right\} .
$$

Then $\|\gamma\|_{\sigma}<1$. Because of (3.1), it suffices to show that

$$
|\gamma(y)-\gamma(x)|<\sigma(|y-x|)
$$

whenever $x, y$ are in $P_{1} \cup\left\{x_{j}^{*}\right\} \cup\left\{x_{i}: i \in N(j+1, q)\right\}$ and $x \neq y$. And (3.8) holds since (j.4) and (3.7) hold, and

$$
\begin{aligned}
\max \left[\left\{\psi\left(y_{i}\right)-\sigma\left(x_{j}^{*}-y_{i}\right)\right.\right. & : i \in N(p)\} \\
\cup & \left.\left\{\varphi\left(x_{i}\right)-\sigma\left(x_{i}-x_{j}^{*}\right): i \in N(j+1, q)\right\}\right]<\varphi\left(x_{j}^{*}\right) .
\end{aligned}
$$

Now let $d^{\prime}$ be a number such that $0<d^{\prime}<\frac{1}{2} \min \left((n q)^{-1}, d\right)$ and let $h=$ $\gamma\left(x_{j}+d\right)-\varphi\left(x_{j}+d\right)=2 d^{\prime} d^{-1}\left(a_{j}-\varphi\left(x_{j}^{*}\right)\right)$. Then $h>0$ and, for all $x$ in $\left[x_{j}+d, x_{j+1}-d\right]=I_{j}^{*}$,

$$
\varphi(x)+h \leqq \gamma(x)
$$

By 3.5, with $J=I_{j}^{*}$, there is $\theta_{j}$ in $Q\left(I_{j}^{*}\right)$ satisfying (1)-(4) of 3.5. Note that $\delta\left(\theta_{j}\right)<n^{-1}$. Define $\psi$ on $I_{j}$ by letting

$$
\begin{aligned}
\psi(x) & =\varphi(x)+\theta_{j}(x) & & \text { if } x \in I_{j}^{*}, \\
& =\varphi(x) & & \text { if } x \in I_{j}-I_{j}^{*} .
\end{aligned}
$$

Clearly $(j+1) .1$ and $(j+1) .3$ hold. Next, since (3.6) and (3.7) hold, 
$(j+1) .2$ holds. Now we verify that $(j+1) .4$ holds. It follows from (3.9) and (2) of 3.5 that, for all $x$ in $I_{j}$,

$$
\varphi(x) \leqq \psi(x) \leqq \gamma(x) .
$$

Next, $\left\|\psi \mid I_{j}^{*}\right\|_{\sigma}<1$. For suppose $x, y \in I_{j}^{*}$ and $x \neq y$. Then

$$
\begin{aligned}
|\psi(y)-\psi(x)| & \leqq|\varphi(y)-\varphi(x)|+\left|\theta_{j}(y)-\theta_{j}(x)\right| \\
& \leqq\left\|\varphi \mid I_{j}\right\|_{\sigma} \sigma(|y-x|)+\left\|\theta_{j}\right\|_{\sigma} \sigma(|y-x|) \\
& <4 L \sigma(|y-x|)+(1-4 L) \sigma(|y-x|)=\sigma(|y-x|) .
\end{aligned}
$$

And $\left\|\psi \mid I_{j}\right\|_{\sigma}<1$. For suppose $P_{2}$ is a $\theta_{j}$-partition of $I_{j}^{*}$ and let $P_{3}=P_{2} \cup$ $\left\{x_{j}, x_{j+1}\right\}$. Since $\left\|\psi \mid I_{j}^{*}\right\|_{\sigma}<1$, it suffices to show that (3.8), with $\gamma$ replaced by $\psi$, holds whenever $x, y \in P_{3}, x \neq y$, and either $x \notin P_{2}$ or $y \notin P_{2}$. But, in this case, since (3.10) holds, $|\psi(y)-\psi(x)| \leqq|\gamma(y)-\gamma(x)|<\sigma(|y-x|)$. Now we shall show that $\left\|\lambda_{j+1}\right\|_{\sigma}<1$. Since $\left\|\lambda_{j+1} \mid I_{j}\right\|_{\sigma}<1$, it suffices to show that (3.8), with $\gamma$ replaced $\lambda_{j+1}$, holds whenever $x, y \in[0,1], x<y$, and either $x \notin I$, or $y \notin I_{j}$. Suppose $x \in I_{j}^{\prime}$ and $y \in I_{j}$. Then, since (3.10) holds, $\gamma\left(x_{j}\right) \leqq$ $\lambda_{j+1}(y) \leqq \gamma(y)$. So there is $z$ in $\left(x_{j}, y\right]$ such that $\gamma(z)=\lambda_{j+1}(y)$. Therefore

$$
\left|\lambda_{j+1}(y)-\lambda_{j+1}(x)\right|=|\gamma(z)-\gamma(x)|<\sigma(|z-x|) \leqq \sigma(|y-x|) .
$$

A similar argument applies when $x \in I_{j}$ and $y \in I_{j}^{\prime \prime}$. It remains to show that $(j+1) .5$ holds. To do this, let $A_{j+1}=A_{j} \cup I_{j}^{*}$ and $\eta_{j+1}=\min \left(\eta_{j}, \delta\left(\theta_{j}\right)\right)$. Suppose $x \in I_{j}^{*}$ and that (3.4), with $\theta$ replaced by $\theta_{j}$, holds for all $y$ in $I\left(x, \delta\left(\theta_{j}\right)\right)\left(J\left(x, \delta\left(\theta_{j}\right)\right)\right)$. Then, if $y$ is as specified,

$$
\begin{aligned}
|\psi(y)-\psi(x)| & \geqq\left|\theta_{j+1}(y)-\theta_{j+1}(x)\right|-|\varphi(y)-\varphi(x)| \\
& \geqq 5 L \sigma(|y-x|)-\left\|\varphi \mid I_{j}\right\|_{\sigma} \sigma(|y-x|) \\
& \geqq 5 L \sigma(|y-x|)-4 L \sigma(|y-x|)=L \sigma(|y-x|) .
\end{aligned}
$$

It is clear that after $q$ steps we have the required function $\psi$.

3.7 THEOREM. If $0<M<24^{-1}$, then $U(\sigma, M \sigma)$ is residual in $C(\sigma)$.

Proof. Suppose $M<K<24^{-1}$. Since $C(\sigma)$ is a complete metric space, it suffices to show that:

(1) for each $n$ in $N^{+}$, the interior of $V(\sigma, K, n)$ is dense in $C(\sigma)$, and

(2) $\bigcap\left\{V(\sigma, K, n): n \in N^{+}\right\} \subset U(\sigma, M \sigma)$.

Proof of (1). Suppose $n \in N^{+}$. First, it follows from 3.3 that the set $D=\left\{\varphi \in Q([0,1]):\|\varphi\|_{\sigma}<1\right\}$ is dense in $C(\sigma)$. Fix $L$ in $\left(K, 24^{-1}\right)$. It follows from 3.6 that $V(\sigma, L, n) \cap D$ is dense in $D$. We shall show that $V(\sigma, L, n)$ is contained in the interior of $V(\sigma, K, n)$. Suppose $f \in$ $V(\sigma, L, n, \eta)$. Let $d=\frac{1}{2}(L-K) \sigma\left(\frac{1}{4} \eta\right)$. We shall show that

$$
\{g \in C(\sigma):\|f-g\| \leqq d\} \subset V(\sigma, K, n, \eta) .
$$


There is a measurable subset $A(f)$ of $[0,1]$ such that $m(A(f))>1-n^{-1}$ and, if $x \in A(f)$, then there is $\delta(x, f)$ such that $\eta<\delta(x, f)<n^{-1}$ and (3.5) holds either for all $y$ in $I(x, \delta(x, f))$ or for all $y$ in $J(x, \delta(x, f))$. Suppose $g \in C(\sigma)$ and $\|f-g\| \leqq d$. Let $A(g)=A(f)$ and, for $x$ in $A(g)$, let $\delta(x, g)=$ $\delta(x, f)$. If (3.5) holds for all $y$ in $I(x, \delta(x, f))$ and $y \in I(x, \delta(x, g))$, then, since $\sigma(|y-x|) \geqq \sigma\left(\frac{1}{4} \eta\right)$,

$$
\begin{aligned}
|g(y)-g(x)| & \geqq|f(y)-f(x)|-2 d \\
& \geqq L \sigma(|y-x|)-(L-K) \sigma(|y-x|)=K \sigma(|y-x|) .
\end{aligned}
$$

Similarily, if (3.5) holds for all $y$ in $J(x, \delta(x, f))$, then (3.12) holds for all $y$ in $J(x, \delta(x, g))$. Hence $(3.11)$ holds.

Proof of (2). Suppose $f \in \bigcap\left\{V(\sigma, K, n): n \in N^{+}\right\}$. Then there is a sequence $\left(A_{n}\right)_{n \in N^{+}}$of measurable subsets of $[0,1]$ such that $\lim _{n \rightarrow \infty} m\left(A_{n}\right)=$ 1 and, if $x \in A_{n}$, then there is $\delta(x, n)>0$ such that $\delta(x, n)<n^{-1}$ and (3.5), with $L$ replaced by $K$, holds either for all $y$ in $I(x, \delta(x, n))$ or for all $y$ in $J(x, \delta(x, n))$. Let

$$
A=\bigcap\left\{\bigcup\left\{A_{k}: k \in N^{+}, k \geqq n\right\}: n \in N^{+}\right\} .
$$

Then $m(A)=1$.

Suppose, now, that $g \in C(M \sigma)$ and let $X=\{x: f(x)=g(x)\}$. Suppose $x \in A \cap X$. We shall show that the lower metric density of $X$ at $x$ is $<1$. There is an increasing sequence $(k(n))_{n \in N^{+}}$of positive integers such that $x \in \bigcap\left\{A_{k(n)}: n \in N^{+}\right\}$. For $n$ in $N^{+}$, let

If $n \in N^{+}$, then

$$
I_{n}=\left[x-\frac{1}{2} \delta(x, k(n)), x+\frac{1}{2} \delta(x, k(n))\right] .
$$

$$
m\left(\{y:|f(y)-f(x)| \geqq K \sigma(|y-x|)\} \cap I_{n}\right) \geqq \frac{1}{4} m\left(I_{n}\right) .
$$

Since

$$
\{y:|f(y)-f(x)| \geqq K \sigma(|y-x|)\} \subset\{y: f(y) \neq g(y)\},
$$

$4 m\left(X \cap I_{n}\right) \leqq 3 m\left(I_{n}\right)$. And, $\lim _{n \rightarrow \infty} m\left(I_{n}\right)=\lim _{n \rightarrow \infty} \delta(x, k(n))=0$. Thus the lower metric density of $X$ at $x$ is $\leqq 3$. Hence, by the Lebesgue density theorem, $m(A \cap X)=0$ and $f \in U(\sigma, M \sigma)$.

ProOF OF THEOREM 1.2. Choose $M$ so that

$$
\limsup _{x \rightarrow 0}\left[\mu(x) \sigma^{-1}(x)\right]<M<24^{-1} .
$$

We shall show that $U(\sigma, M \sigma) \subset U(\sigma, \mu)$. First, there is $\delta>0$ such that $\mu(x) \leqq M \sigma(x)$ whenever $0 \leqq x \leqq \delta$. Suppose $f \in U(\sigma, M \sigma), g \in C(\mu)$, and $J$ is a closed subinterval of $[0,1]$ of length $\leqq \delta$. Then $g \mid J \in C(J, M \sigma)$. Let $h$ in $C(M \sigma)$ be such that $h|J=g| J$. Then $m(\{x: f(x)=h(x)\})=0$ since $f \in$ $U(\sigma, M \sigma)$; hence $m(\{x: f(x)=g(x)\} \cap J)=0$. Therefore $f \in U(\sigma, \mu)$. 
3.8 THEOREM. Let $V(\sigma)$ denote the set of all $f$ in $C(\sigma)$ such that $m(\{x: f(x)=g(x)\})=0$ for all $g$ in $\bigcup\left\{\operatorname{Lip}(\mu): \lim _{x \rightarrow 0}\left[\mu(x) \sigma^{-1}(x)\right]=0\right\}$. Then $V(\sigma)$ is residual in $C(\sigma)$.

PROOF. If $0<M<24^{-1}$ and $\lim _{x \rightarrow 0}\left[\mu(x) \sigma^{-1}(x)\right]=0$, then $U(\sigma, M \sigma) \subset$ $\bigcap\{U(\sigma, K \mu): K>0\}$. Hence $U(\sigma, M \sigma) \subset V(\sigma)$.

3.9 Definition. For $\alpha$ in $(0,1]$, let $\sigma_{\alpha}(t)=t^{\alpha}$ for all $t$ in $[0,1]$. For any subset $A$ of $[0,1]$, let $\operatorname{Lip}_{\alpha}(A)=\operatorname{Lip}\left(A, \sigma_{\alpha}\right)$. Denote $\operatorname{Lip}_{\alpha}([0,1])$ by $\operatorname{Lip}_{\alpha}$.

In the rest of this paper $\alpha$ will always denote a number in $(0,1)$.

3.10 Corollary. Suppose $M>0$. Let $W(\alpha, M)$ be the set of all $f$ in $C\left(M \sigma_{\alpha}\right)$ such that $m(\{x: f(x)=g(x)\})=0$ for all $g$ in $\bigcup\left\{\operatorname{Lip}_{\beta}: \alpha<\beta \leqq 1\right\}$. Then $W(\alpha, M)$ is residual in $C\left(M \sigma_{\alpha}\right)$.

ProOF. This follows from 3.8 since $M \sigma_{\alpha}$ is a concave modulus of continuity.

3.11 THEOREM. Suppose $\mu$ is a subadditive modulus of continuity, $A \subset[0,1]$, and $f \in C(A, \mu)$. Then there is $f^{*}$ in $C(\mu)$ such that $f^{*} \mid A=f$.

The proof of 3.11 is the same as in the special case where $\mu=M \sigma_{\beta}$ for some $\beta \in(0,1]$ and $M>0$.

3.12 Corollary. Suppose $\sigma, \mu$ satisfy the hypothesis of 1.2. If $f \in U(\sigma, \mu)$ and $A$ is a subset of $[0,1]$ such that $f \mid A \in C(A, \mu)$, then $m(A)=$ 0 .

ProOF. This follows from 1.2 and 3.11 , since any concave modulus of continuity is subadditive.

In [2], W. S. Loud constructed a function $f$ in $\operatorname{Lip}_{\alpha}$ such that, if $\alpha<\beta \leqq 1$ and $J$ is a closed subinterval of $[0,1]$, then $f \mid J \notin \operatorname{Lip}_{\beta}(J)$. The following statement shows that, in a certain sense, most functions in $\operatorname{Lip}_{\alpha}$ have a somewhat stronger property.

3.13 Corollary. Suppose $M>0$. Let $W(\alpha, M)$ denote the set defined in 3.10. If $f \in W(\alpha, M)$ and $A$ is a subset of $[0,1]$ such that $f \mid A \in \bigcup\left\{\operatorname{Lip}_{\beta}(A)\right.$ : $\alpha<\beta \leqq 1\}$, then $m(A)=0$.

Denote the set of all real numbers by $R$. In [2], W. S. Loud constructed a function $f: R \rightarrow R$ with the following property: There are positive numbers $M, K$ such that $K<M$ for which $|f(y)-f(x)| \leqq M|y-x|^{\alpha}$ for all $x, y$ in $R$ and

for all $x$ in $R$.

$$
\limsup _{h \rightarrow 0}\left|[f(x+h)-f(x)] h^{-\alpha}\right| \geqq K
$$

3.14 Definition. Suppose $F$ is a measurable function defined on $(-a, 0) \cup(0, a)$ for some $a>0$. The approximate upper limit of $F$ at 0 , 
denoted by lim sup ap $\operatorname{ap}_{x \rightarrow 0} F(x)$ is defined to be the infimum of the set of real numbers $y$ such that $\{x: F(x) \leqq y\}$ has metric density 1 at 0 .

Note that lim sup ap $\operatorname{ap}_{x \rightarrow 0} F(x) \leqq \lim \sup _{x \rightarrow 0} F(x)$.

3.15 Corollary. Suppose $0<K<24^{-1} M$. Let $S(\alpha, M, K)$ denote the set of all functions $f$ in $C\left(M \sigma_{\alpha}\right)$ such that

$$
\lim \sup _{h \rightarrow 0} \text { ap }\left|[f(x+h)-f(x)] h^{-\alpha}\right| \geqq K
$$

for almost all $x$ in $[0,1]$. Then $S(\alpha, M, K)$ is residual in $C\left(M \sigma_{\alpha}\right)$.

Proof. This follows from the proof of 3.7, since

$$
\bigcap\left\{V\left(M \sigma_{\alpha}, K M^{-1}, n\right): n \in N^{+}\right\} \subset S(\alpha, M, K) .
$$

\section{ReFERENCES}

1. C. Goffman, Approximation of non-parametric surfaces of finite area, J. Math. Mech. 12 (1963), 737-745. MR 27 \#3782.

2. W. S. Loud, Functions with prescribed Lipschitz conditions, Proc. Amer. Math. Soc. 2 (1951), 358-360. MR 13, 218.

Current address: 251 N. Blackburn Road, Rt. 5, Athens, Ohio 45701 